\title{
TWUNEEFS
}

PERICUhUK BNTOMOLOGICUM

$$
\text { Quo }
$$

\author{
CHAPACTERES
}

OENERUM INEECTORUN

\author{
1729
}




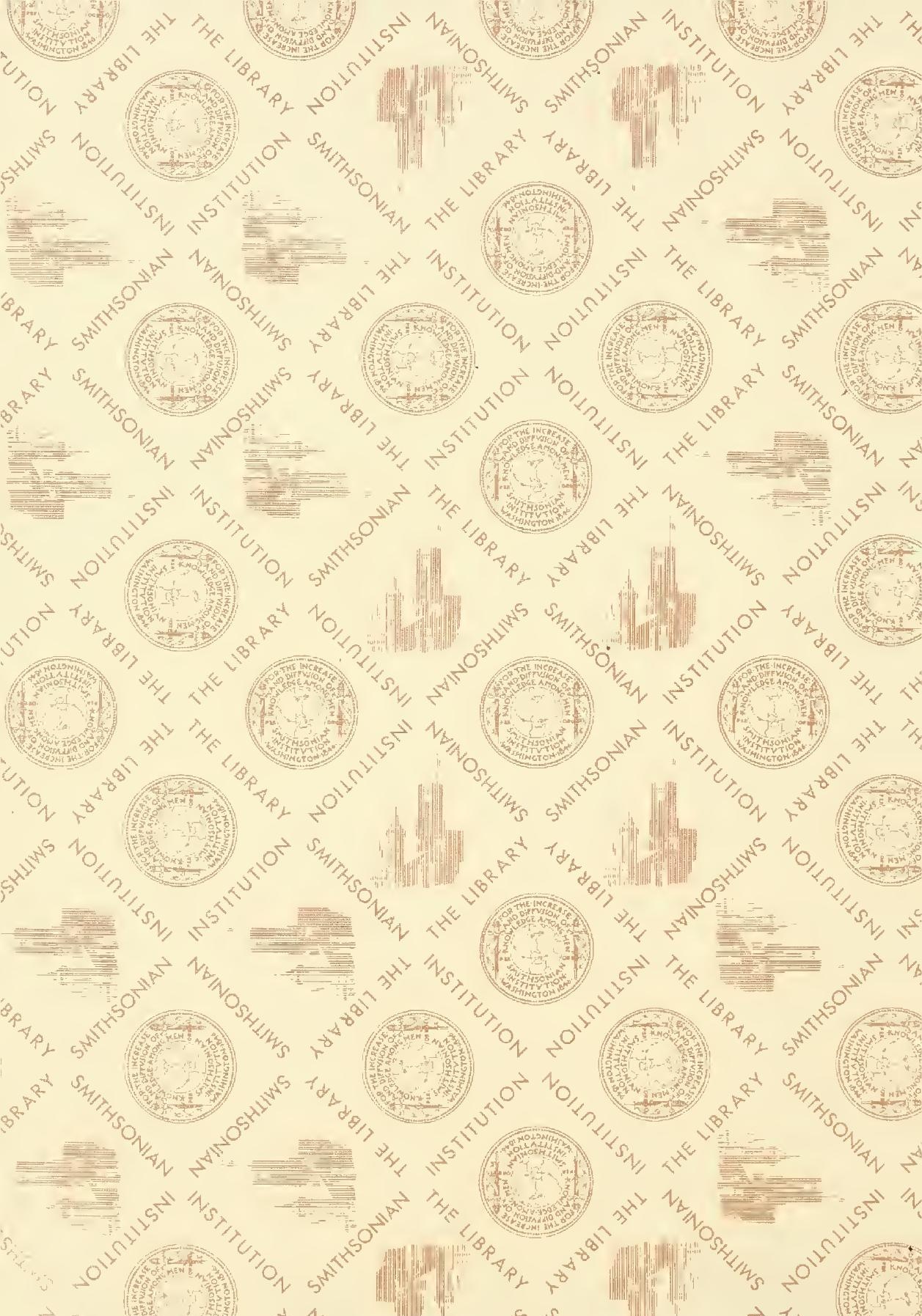





\section{CHARA CTERES}

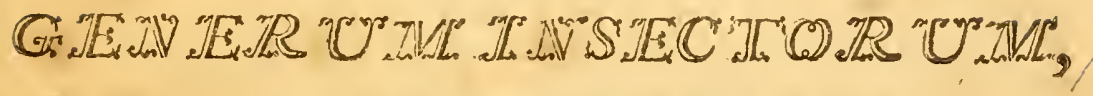
=5?

CONSENSU EXPER. FAC. MED. UPSAL.

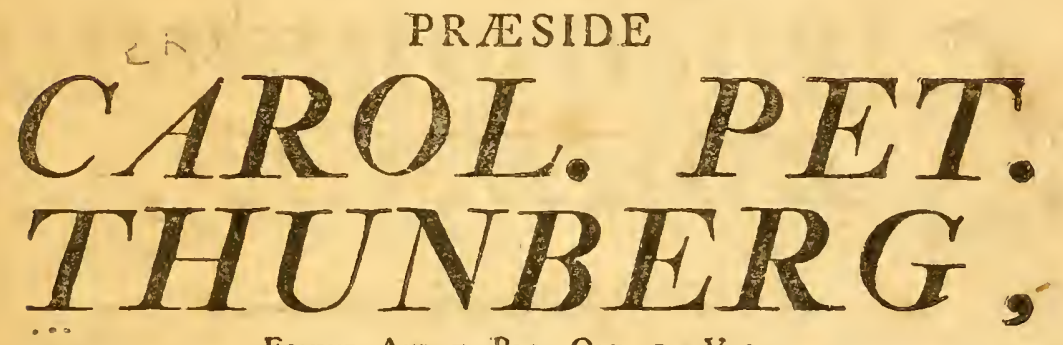

Equite Aurat. Reg. Ord. de Vasa.

Medic. Doct. Profess. Med. et Botan. Reg. et Ord., Acad. Cessar. Nat. Curios, Rig. Scient, Londinens. Holmiens, Societ. Scient, Lipsat. Patr. Hol m. Berol. Nat. Scrut. lundens. Harlem. Amsteld. Zes.and. Nidrosiens, Halens. Nat. Scrut. Medic. Edimburg. et Nat. Studios. Ibid。 Membro; nec non acad Scient. Paris. Monspeldens. Agricult. Paris. Florentin. et batavin. Ind. Or. Correspondo

PUBLICE CENSURÆ PROPONIT

$$
\text { SAMUEL } \underset{\text { Norcopia.Gothus. }}{T O B R N E R,}
$$

IN AUDIT. GUSTAV. MAJ. D. X JUN. MDCCLXXXIX, Horis confuetis.

UPSALIE, LITIERIS DIRECTOR. JOH. EDMAN.

$$
1789 .
$$

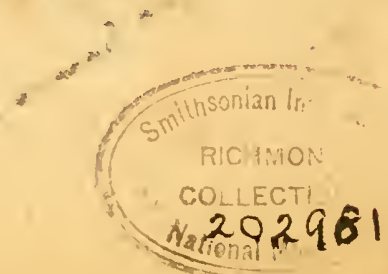


IN S:AM R:AM $M: T E M$

\section{SPECTATA FIDEI VIRO,}

AD STATUM FORTIFICATIONIS

\section{CAPITANEO,}

\section{GEIVEROSO AC NOBILISSIMO}

\section{DO $\mathscr{T} \mathscr{J} \mathscr{N O}$}

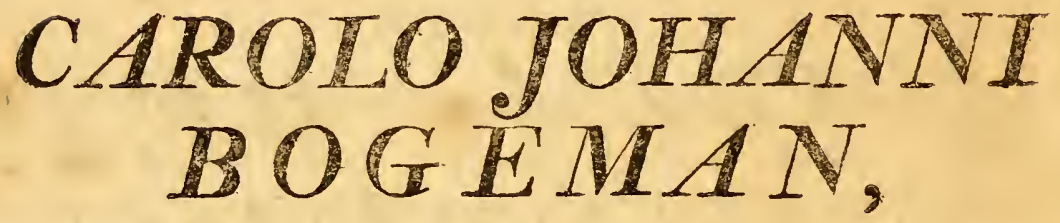

FAUTORI PROPENSISSTMO.

Hximiam, qus polles Hiftoria Natmalis peritiam, \& fum Le man, qua ex illa colenda perfruiris, voluptatem fuspici. ens, fovoremque, quo me anplecii dignatus es, votis mis mevicisyue lunge nsajorem, grato recolers asimo, $T E$ bard agre laturum credidi, guod bas pagellas TIBI biumillinse aticare audenm, funna, donec vixero, mentis veneratione per. manjuras

GENEROSI AC NOBLLISSIMI PJOMLNIS TUI

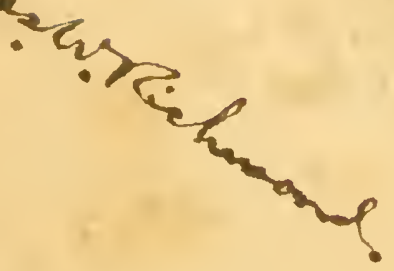

cultor bumillimns

SAMUEL TÖRNER。 


\section{Prooeminn.}

Eam, utique deprehendimus Syfematum in gratiam Entomologice concinnatorum copiam, ut plura ejusdem generis molimina fupervacanea videantur. Sic firenuam Eo felici cum fuccesfu in illa excolenda at que ad faftigium perducenda operam collocarunt bene multi Syftematici eo directam, ut ad naturce feetus forutandos facilior panderetur aditus. Sed in hoc prreftando in diverfa abierunt Auktores, dispari modo fciendi cupidis fatisfacientes. Quos inclitos fcientice Conditores maximopere Jupereminet Hiftoria Natura lis ftator immortalis a LINNé; qui Summus Vir, ut ob Sparfa lumina per reliquas Hiftorice ejusdem partes incomparabilis, fic etiam ex Infectis Syftematica ferie tradendis nihil non glorice reportas $\int_{e}$ dicendus eft. Grato ac perspicuo proponendi more je ubique comsnendat hujus ingeniofisfimi Vir imethodus, natura curiofos in $\int u$ amorem ac admirationem vi quadam ipfo propria rapiens.

Hic etiam fllentio neutiquam pratermittere posfumus de re Entomologica longe Meritisfimi Prof: FABRICII indefesfan Eo eximiam plane operam, qui novum condendo Syfema, Entomologorum in Se cons veritit oculos, E' cujus Scripta celeberrima omnium corum jam verfantur manibus. Maxime fane confentaneum omnium foret votis, ut hunc incredibili preditum perfpicacia Virum in fubtilisfmis insectorum A 3

par. 
partibus quisque asfequi, E ipfus Charafteres Genericos eruere valevet. Cum vero ea fit oporteat hujus amoence Scientice indoles, ut ejus principia, quoad fiere posfit, perveftigatu facili methodo propofita, in omnium Sefe infinuent oculos cultoram, vereor ne po. tius nimice curiofitati fatisfacienda inferviat labor, in inftrumentis cibariis infectorum, fecundum methodum Fabricianam, adeo ad unguem refecandis, collocatus, quam intento refpondeat usui; atque Scientice cultores Nomenclature fupra laudati Viri magis adhereant, habitum infectorum in ejus operibus traditorum ex Synonymis Linnceanis pernofcentes, quan notas Gemericas ipfius syflematis irrito perquirere nitantur. N'os igitur Syflema Scrutatcrum captui accommodatum exigendo, caftra Fabriciani deferere cogimur, charabteres generibus infectorum ab ipfo impofitos, in minoribus pracipue, haud diffciles molo, fed esfe inextricabiles plane perfvafi: proinde aliis, quibus volupe erit, arue lynceis nature Scrutatoribus mandibulas ac maxillas infectorum earumque fitum Eo dentes eruenda relingwimus, contenti, $f$ in externis, aut magis confpicuis haud deftruendi animalis partibus notas quas. dam detegere valemus horum animantium familiis difcer. nendis applicatas. Quo in negotio, fi LiNNAI veftigia legentes $a b$ iftis nos interdum defleciere offendimur, id mirum non erit perpendenti, tot esfe infectorum fpecies poft tempora ipfius detectas, ut ad fingulas inveniendas Eo rite cognofcendas opus fit illas in plura genera dis. jungere; cujus rei necesfitatem adeo quemvis perfoicere convincimur, ut omnem demonftrationem fuperfluam. ducamus. 
Ad ordines infectorum quod attinet, illos, quos fto. tuit a LINNé, integros Servamus, divortia Sententiarum cum F ABRIClo facientes, qui Apem, Ephemeram Es Onifcum fub eadem clasfe confociat, qui Libellulam E' Araneam fimili modo conjungit, E' Acarum ac Oeftrum fub eodem ordine proponit.

Difficultate ergo in lifce mire variantibus animalcu. lis dignofcendis penfitata, Celeber. D. PRASES, pro fuo in Hifforice Nat. incrementa ardore de eo fuit follicitus, ut Entomologorum labor hac in re quodammodo fubleva. retur; quem in finem prefens opufculum corum propo. nitur difcrimini, quo paco fciendi cupidis methodus, qua in Museo Acad. Upfal. adornando ufus fit, cum illis emendationibus, quas, poft illius jam editum Catalogum, iteratce ob fervationes inflituendas fvaferunt, fimul inno. tefcat.

Editurus Specimen guoddan Academicum rogavi, us vires in illo negotio conficiendo mihi periclitari permit. teret ilem Vir Celeberrimus: Quamobrem hos Chara. cieres infecforum qualescunque Tibi B. L. fiftit temuis mei ingenii fuppellex, indulgentem juvenilium conatuum: sxcufationem enixe mili expetens. 


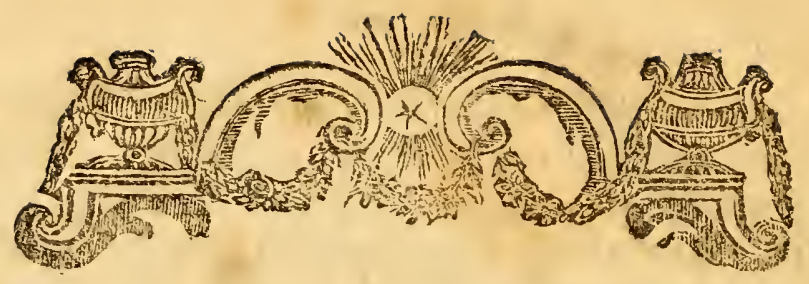

- Fag med Linné berragear

Nacureis usdransvấda fpar. Paykete.

\section{Coleoptera.}

* Antennis clava folida.

Pausus: Clava uncinata. Elytra abbreviata. Hister: Caput recraetum. Elytra abbreviata. Coccinerla: Corpus hemisphrericum, fubrus planum. Anthrenus: a) Caput retractum. Thorax ancice coardacus. Corpus ovatum.

Elorhorus: Thorax quadratus. Elytra æquantia.

\section{Bulbocerus:}

\section{** Antennis clava bulbiformi.}

\section{*** Antennis clava lamellaza.}

Lucanus: Maxillæ porredx.

Scarabeus: b) Caput exruberans. Tergum (cutellatum. Copris: Caput extuberans. Tergum exfutellatum. Melolontha: Caput muticum. Trox: Caput retractum. Abdomen fubtus planum.

a) Sub Byrshi nomine comprehenfus in Cat. Muf. Upr.

b) Hoc, ut fequens genus, thosacem induit elytris adsquetum, ampliato-productum, fed Melolonthe thoraz eft elyeris inadaquatus, anguttatus, brevis. 
***** Anrennis clava perfoliata.

Curcuno: c) Caput roftratum, poftice incrasfatum. Atrelabus: Caput roftratum, poftice atrenuarum. Hydrophilus : d) Corpus ovale, fornicatum. Pedes natatorii. Spharidium: e) Corpus ovatum, fornicatum. Pedes gresforii. Byrrhus: $f$ ) Corpus ovatum, convexum. Thoray antice coarctatus.

Dermestes: Corpus oblongum, depresfufculum. Thorax ancice attenuato-coarctatus.

Bostrichus $: g$ ) Caput retraktum. Thorax teres, Corpus cylindricum.

Anobrum: b) Corpus lineari-oblongum. Caput deffexum. Eilytra flexilia.

Tritoma: 2) Corpus lineare, depresfum. Thorax quadratus. IPs: k) Corpus ovato-oblongum, undique convexum. Silpya: 1) Thorax Elytraque lateribus explanatis.

Nitidula: ms) Thorax transver(us. Elytra truncata.

Necrophorus: n) Thorax rotundatus, lateribus explanatis. Elytra plana, truncata.

****** Antennis moniliformibus.

Opatrum: o) Thorax convexus, antice lunatus, lateribus explanatis. Elytra convexa.

BRU-

c) Antenna infidentes roffro, ob oculis remota. (d) Hydrous Cat. Mof. Upf. e) Cospus ad hemifpharican figuram accedit in plurimis. Thorax ampliatns. f) Ingreditur Spharidii genus in Car, Muf. Upto g) Ips Car. Muf. b) Obf. Caput thoraci fubaquale. Thorax rotundssus. Antennx in quibusdan Speciebus monilifnrmes. i) Cum hoc chia. satere conveniunt Dermeftes ferrugineus, Tritoms colon. Obf. Caput exfertun. k) Genus Fabr. ad quod pertinet Ips anea: hac quociue referinus Silphan rusfican in Cat. Mur. l) Corpus depresfiufculum, ovatum. Caput retrasto-infexum. Antenna moniliformes in multis. m) Co:pus quadrarun. n) Hoc genus conftitument Nitidula litroralis \& $N$. vefpillo Car. Mnl. Unt. Nicroph. in feripris Fabr. fphalma forte eft. a) Huic asfociantus Silpha cornuta, Hifpa muica \& Opatrum Fabr. 
Bruchus: Caput rerracto-inflexum. Thorax antice anguftatus. Elytra obtula.

Hrspa : p) Thorax antice anguftior, immarginatus. Elytra obeufa.

Cassida: Corpus convexum, fubtus planum. Thoracis clypeus caput obtegens.

Chrysomela : Corpus ovatum. Thorax transverfus, mar. ginatus. Elytra immarginata.

Altica: Pedes Caltatorii.

Mordella: Corpus incurvatum. Elytra attenuata. Lamina ante femora.

Erodius: Thorax transverfus, depresfus. Abdomen fub. globofum.

Mezoë: q) Capur inflexum. Thorax lateribus adpresfus, fubrotundus. Elytra linearia, flexilia.

Clerus: Caput thorace latius, refimum. Thorax poftice attenuatus.

Spondylis: $r$ ) Thorax elytris anguftior. Corpus fubcylin. dricum.

Prmela : s) Thorax rotundatus, convexus, marginatus. EJytra convexa, bimarginara.

Tenebrio: $t$ ) Thorax antice lunatus, elytris fubrqualis, marginatus. Corpus oblongum.

Staphylinus: $u$ ) Elytra abbreviata. Cauda bifeta.

****** Antennis filiformibus.

Grrinus: Antennæ cylindricæ, capite breviores. Oculi quatuor. Noto.

p) Anpennz porrętr, approximatx. Corpos fropius aculeatun. 9) Caput grnnd, retufum. r) Ad hoc genus referimus Tenebrionem afperum, Fpond. bupreftoiden, quamvis antennx non nihil abludant. s) Difficillime nb hoc genere dislociari queunt Sepidii plurimx Species in Cat. Muf., hinc combihavimus. Sepid. vituntum Erodii intrat gents. 8) Thorax fere quadrarus. u) Corpus lineare. Ahdominis margo ele. varue. Veficule 2 fupra caudam exferends. Ale elyrris obrectx. 
Noroxus: Thorax gibbus, utrinque attenuatus:

Prinus: Caput retracto inflexum. Thorax antice coardtatus. Melyris: Caput inflexum. Thorax bimarginatus.

CrIoceris: Antennæ approximatæ. Thorex fubquadratus. Elytra oblonga.

Cryptocephalus: Caput retufo-retractum. Elytra obtuf. Corpus cylindricum.

Avchenia: $x$ ) Thorax teres, elytris depresfiufculis anguftior.

Evrychora: y) Thorax fubquadratus, depresfus, margine furfum dilatato. Elyrra depresfa.

Cucujus: Corpus depresfum. Thorax poftice rotundatus. Buprestis: z) Caput thoraci (emi-intrufum. Elater: Pectoris mucro e poro abdominis refiliens.

$$
\text { ****** Antennis fetaceis. }
$$

Scarites: a) Thorax lunatus, poftice rotundatus. Carabus: Thorax obcordatus, poftice truncatus, marginatus. Cicindela: Oculi prominuli. Thorax inæqualis. Donacia : b) Thorax quadratus, immarginarus. Cistela: c) Corpus oblongum, fornicatum. Thorax marginatus, antice anguftior.

Dytiscus: Thorax transverfus. Pedes natatorii. Leptura: Thorax fubglobolus, muticus, antice anguftior. Callidium: d) Thorax rotundatus, muticus.

SAPER-

*) Sub hac adpellatione compleßimur Crinceridis generi quasdam fpecies antea injunctas e. g. Cr. merdigeram, cyanellam. y) Ad hoe genus amandamus Pimeliam ciliatam (Cat. Muf.) z) Obf. Oculi in Bupreftide fere oblonsi, in Elatere rotundi. n) Carabus maxillofus. Tenebrio fosfor \&c. hoc conftituunt genus. b) Habitus nobis perfvafit esfe Calopum aquaticum, Spondyl. femoratum (Cat. Muf.) heic conjun. gendas. c) Heic in confiderationem veniunt Crioceris fulphurea, C. ceramboides, fimilescjue in Car. Muf. sulticum, \& hujusmodi in LxnNal Syßtem. 
SAPERDA: e) Thorax cylindricus, muticus.

Prionus: $f$ ) Thorax planus, margine acuto, crenulatoEpinolo.

Cerambyx: *) Thorax lateribus extuberanti-fpinolus.

Calopus: g) Thorax teres, elytris anguftior. Corpus cy. lindricum.

Cantharis: Caput exfertum. Clypeus thoracis planus, ely. tris æqualis. Elytra linearia, flexilia.

Pyrochroa: b) Caput exfertum. Thorax rotundatus. Elytra flexilia. Corpus poftice incrasfatum.

Calolymus: i) Caput globofum. Thorax antice coarctatlis. Elytra linearia, flexilia.

Malachius: Elytra attenuato-anguftata.

Lampyris: Caput tectum clypeo thoracis. Elytra flexilia.

Necrdalis: k) Elytra abbreviata. Cauda fimplex. Forricula: Elytra abbreviata. Cauda forcipata.

II. HEM:-

e) e. g. carcharias (Ceramh. Linn.) f) e. g. Cerambyx co. riaritus Cat. Muf. *)-Viderentur quidem diverfi in Cerambycis genere habirus diverfa prodere genera, modo charakter in his femper conftans \& perfpicuns ohfervaretur. Curioforum ergn vel ficantes defideriis varias hive jus generis facies ira proponimus: Lamis: Thorax teres, fpinofus. Elytra clunata. (i. e. poftice convexa.) Cerambrx: Thorex teres, fpinofus. Elytrs faftigiata, linearia. Obf. faftigiatum fumitur eo lenfu, quo a $L_{1 N}$. mso adhibetur in Bupreft. ruftica. Rhagrum: Caput elongatum. Thorax reres, fuinofus. Elytra fubfaftigiata, cuneara. Stenocorus: Caput elongatum. Thorax teres, extuberans. Elytra cuneata. Adduntur $\mathbf{t u m}$ etiam ceteris adfinibus fequentes notr disjunctive: Calopus: Thorax cylindricus, e. lytris anguftior, muticus. Elytra clunata. SAPERDA: Thorax cylindricus, muticus. Elytra faltigiats. Callidius: Thorax rotundarts. Elytra faltigiate. g) Ab hoc genere non abludunt Cantharis viridisf. \& Calopus ferraticornis. b) Nos, ut Fabricius, lub hac Canthar. pectinicornem Linn. confideramus. i) Lymexylon Fabr. it. Horia. k) Obf. Alax exferrz, nudz; Forficulz teets. 


\section{HEMIPTERA:}

* Antennæ enfiformes. Os maxillis.

Truxalis: Caput conicum, elongatum.

Antennæ filiformes. Os maxillis.

ACRYDIUM: Thorax ultra alas productus.

Gryllus: () Thorax hemelytris brevior. Alæ deflexa.

Pnevmora: Alæ planæ. Corpus inane, inflatum.

** Antennæ feracer. Os maxillis.

Locusta: Alæ deflexæ. Pedes faltatorii.

Acheta: Alæ incumbentes, inferiores caudatæ.

Blatta: m) Pedes curforii : Antici breviores.

Mantis: Pedes antici raptorii, longiores.

Os roltro:

Fulgora: Antennæ capitatæ. Frons producta, inanis. Cicada: $n$ ) Antennæ fubulatæ. Alæ deflexæ.

Membracis: o) Antennæ fubulatæ. Thorax gibbus, poftice attenuato-productus, abdomen adæequans.

Tetrigonia: p) Antennce fubulatæ. Alæ deflexæ. Laminæ ad femora poltica.

Cimex: Antennæ articulatæ. Alæ planæ, cruciatæ, Pedes curforii.

Notonecta: Pedes polteriores natatorii.

NEPA: Pedes anteriores cheliformes, reliqui ambulatorii. ApHIs : *) Antennæ fetaceæ, thorace longiores. Alæ, ubi adfunt, erectx. Pedes ambulatorii.

Chermes: Roftrum pectorale. Antenna retigera. Alx deflexæ. Pedes faltatorii.

Coccus: Roftrum pectorale. Antennæ filiformes, breves.

B 2 THRIPS:

l) Alx lateribus corporis adpresfx, iten in Locufta. m) Thorax orhiculatus. eft generis. infigniuntur.

n) Redes faltatorii。

o) Cicada cornuta Linn. hujus p) Cicodx manniferx, non foltantes (LisN.) heic a nobis -) Abdomen fape poftice bicorne. 
THRIPS: :) Roftrum obfoletum, rima. Alæ incumbentes, fubcruciatæ. Abdomen furfum reflexile.

\section{LEPIDOPTERA.}

Papizio: q) Antennæ apicem verfus crasfiores. Alæ patulæ. SpHinx: r) Antennæ medio incrasfatæ. Alæ deflexæ. Bombyx: s) Antennæ peetinatæ. Lingua recondita. Alx deflexæ, ampliatæ.

NoctuA: Antennæ fetaceæ. Lingua fpiralis. Alæ deflexæ, anguftatx.

(tes.

Phalzena: $t$ ) Antennæ filiformes peEtinarxque. Alre parent Pyralis: $u$ ). Antennæe filiformes. Alæ deflexæ, anguftatæe. Tortrix: Antennæ filiformes. Alæ ampliatæ, obtufæ: margine exteriore curvo.

Tinea : v) Antennæe fetacex. Alæ acutæ, convolutæ. Alucita: Antennæ filiformes. Alæ fisfro

IV. NeVroptera.

Liselzura: Antennæ fubulatæ. Alæ extenfæ, omnes æquales. Cauda hamofo-forcipata.

Agrion: $x$ ) Antennæ fubulate. Oculi extuberantes. Ala erectæ, æquales.

Mrrmaleon: Antennæ capitatæ. Alæ deftexæ, xquales.

EPhemera: Antennæ fubulatæ. Alæ erectæ, pofticæ mini. mæ. Cauda ferofa.

Phryganea: Antennæ fetacer. Thorax brevisfimus. Alax deflexæ.

Heme-

*) Ale dux.

q) Obf. corpus anguftum.

r) Corpus obefum. 8) Autennz maris fxpius pectinatx, feminx interdum fetacex. ) Obf. Ala horizontales. Corpus gracile. Antennz petinatse \& feracex in di. Atinto lexu. u) Alx conniventes in figursm deltoideam, v) Alx convoluta in formem cylindricam. $x$ ) Genus Fabr. Oculi prominuli e, g. LibelIula puello L1\%k. 
HEMERobrus: Antennæ fetaceæ. Thorax rotundatus. Alæ deflexæ.

Panorpa: Antennæ fetaceæ. Os roftro corneo, cylindrico. Alæ incumbentes.

Raphidia : Antennæ fetaceæ. Caput triangulare. Thorax elongatus.

Termes: Antenna moniliformes.

\section{Hymenoptera.}

Formica: a) Antennæ medio geniculatæ. Petiolus abdominis extuberans.

Mutilla: b) Antennæe extrorfum attenuatæ. Os elingue. Petiolus abdominis æqualis. Corpus totum $\mathrm{pu}=$ befcens.

Aprs: c) Lingua inflexa. Alæ planæ.

VesPA: d) Antennæ extrorfum crasfiores. Alæ fuperio. res plicatæ.

Sphex: e) Antennæe extrorfum attenuatæ. Alé planæ, fubincumbentes.

CHrysis: $f$ ) Abdomen fubtus fornicatum, utrinque fqua: ma laterali. Aculeus fubexfertus.

Sirex: g) Abdomen fesfile, mucronatum. Aculeus exfertus fub adominis Spina terminali.

ConxnIs: b) Antennx capitatx. Abdomen fornicarum.

TENa

a) Antenna filiformes, fracta. Oculi minuti. Als plana incumbenres, neutris nullx. Aculeus obfolerus. b) Alx plana, feminis nulla. Abdominis primum fegmentun maximum. Aculeus punetorius. c) Antenna filiformes. Oculi oblongi. Aculeus fermins \& neutris panctorius. d) Antenuz pendula. Os elingue. Oculi reniformes. e) Ancenna incur. va. Oculi nvari. Aculeus punctorius. f) Os elingue. Ala planz. Cor. pus fulgidum. g) Anrenna feracer. Thorax antice lunatus. b) Sub hac denominatione innuimus Tenthredinem luteam, obfcuram, \& hifce fimiles, gux alias jisdem notis infigniuneur, ac Genus infequens, Tenturedo. 
Tentredo: i) Antennæ fubfiliformes. Abdomen resfile; oblongum. Aculeus ferratus, bivalvis, reconditus. ICHNEvmon: Antennæ fetacer. Abdomen petiolatum, elongatum. Aculeus exfertus, bivaginatus.

Cynips: k) Antennæ filiformes. Abdomen fubovale, compresfum. Aculeus fpiralis.

\section{Diptera.}

Oesteus: Os claufum, poro pertufum. Anrennæ globo. fe, fetarix.

Stratromys: l) Proborcis proclinata. Antennæ lanceola. tæ, bafi conniventes. Alæ incumbentes. Scutelium dentatum. Abdomen rotundum.

Biblo: $m$ ) Probofcis proclinata. Antenna filatæ, acuminatæ. Caput globolum. Alæ extenfæ.

Musca: n) Probolcis proclinata. Anrenna incrasfatx. breves. Scutellum muticum.

Rhagro: o) Probofcis exferta, palpis incumbentibus. Antennæ fubulatæ. Abdomen fubconicum.

Tabanus: p) Probofcis exferta, palpis incumbentibus. Antennæ filatæ: Abdomen ovale.

Trpula: Probofcis brevisfima. Palpi duo, porrecti, incurvi. Antennæ filiformes.

i) Thorax huic antice æquali-obtufus. k) Larvæ causlæ font Gal. Jarum. () Huic generi inferimus Mufcam musbilem Linn. \& $\mathrm{M}$. chamal. cum reliquis iftius ftruekura, m) Hoc loco denotamus Mufcam mauran L. \& huic fimiles, cum charaetere convenientes. (n) Sub hoc genere combinavimus Mufcam \& Syrphun Fabr. Alias fxpisfine dignofcitur Syrphus Corpore tomentofo, aut glabro, depili. (i. e. nullis

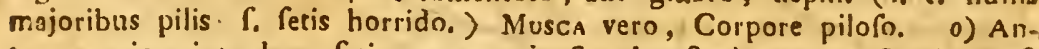
tenna apice interdum fetigerx, ut in Syrpho Scolopacen, Catal. Mur. A. Upf. Thorax huic generi gibbus. Corpus elongatum. Genus eft Fabr. cujus Gener. habitum fequimur. p) Oculi transverfales in plurimis, fed. in Musers, Stratiom. Rhagion. longitudinales, vel rotundi, Probolcis TaBasi aculeis compresfis, acumiratis predita eft. 
Conops: Probolcis geniculata, fetiformis, longa. Antennæ clavato-acuminatæ. Abdomen clavatum, in. flexum.

Curex: Hauftellum elongatum: vagina aculeos fetaceos gerente. Antennæ filiformes.

EMPIS: Hauftellum inflexum. Antennæ fubulatæ. Caput globolum.

STomoxys: १) Hauftellum porrectum, fetiforme. Antennæ fetariæ.

Bombyuius: r) Haultellum porrectum, longisfimum. Antennæ fubulatæ.

Asıus: s) Hauftellum porrectum, rectum, corneum. Antennæ fubulatæ.

Hipровоsса: t) Hauftellum breve, cylindricun, nutans.

\section{VII. Áptera.}

Lepisma: u) Pedes VI. Antennæ letaceæ. Cauda trifera. Poduka: v) Pedes VI Antennæ feraceæ. Cauda bifurca. Pediculus: $x$ ) Pedes VI. ambulatorii. Antennæ moniliformes. Pulex: Pedes VI, poftici faltatorii. Probolcis brevis, incurva. Abdomen compresfum.

Acarus: Pedes VIII. Oculi II. Tentacula II, pediformia. Aranea: Pedes VIII. Oculi VIII. Antennæ nullæ. Os forcipatum. Palpi articulati, incurvi.

Phalangium: Pedes ViII. Oculi IV. Antennz nuliæ. Cad put antice cheliferum.

SCOR=

9) Proponimus hic Afilum Morion. Linn. ()bf. Probofcis in canaJem retrakilis in Conope, Stratiomy, Bibione, Mufca, r) Caput depres. f $m$ Corpus ovale. Alx extenfa. s) Caput compresfum. Frons barba. to. Tharax gibbus. t) Pedes unguiculis pluribus. $\boldsymbol{u})$ Pedes curfo. rii. Cauda teris exrenfis, Corpus fquamarum. v) Pedes curforii. Cauda inflexa, falrarrix, 2 ) Os aculeo rerrsctili, Abdomen depresfom, Sublobatum. 
Scorpro: y) Pedes VIII. Antennæ nullæ. Manus chelatæ. Cancer: Pedes. X. Antenna breves. Corpus rotundatum. Cauda recurvato-recondita.

Pagurus: Pedes X. z) Aurennæ IV, inæquales. Corpus elongatum. Cauda tenui membrana obducta.

Astacus: Pedes X. Antennæ IV, elongatæ. Manus chelata. Corpus elongatum. Cauda extenfa, foliacea. Gammarus: *) Pedes X. Antennæ anticæ breviores. Cauda compresfa.

Monoculus : Pedes XII, plurimi chelati. Antennæ fisfæ. Corpus cruttie innatum.

Oniscus: Pedes XIV. Corpus convexiufculum. SCOlopendra: Pedes totidem utrinque, $q$ quor corporis fegmenta. Corpus lineare, depresfum. Antenпæ fetaceæ.

Julus: Pedes duplo utrinque plures, quam corporis fegmenta. Antennæ moniliformes, Corpus ferni-cy-
lindricum.

y) In plurimis hajus Generis Speciehus oblervatur cauda articulata, mucrone incurvo inftructa. PeEtines II. pedes pofticos adjacent. z) Species in hujus generis Parasitic funt, cauda aphylla diftinetr. *) Huic generi adferibitur Cancer pulex Liño.

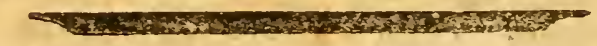

Admiranda tibi levium Spectacula rerum.

VIRG. 



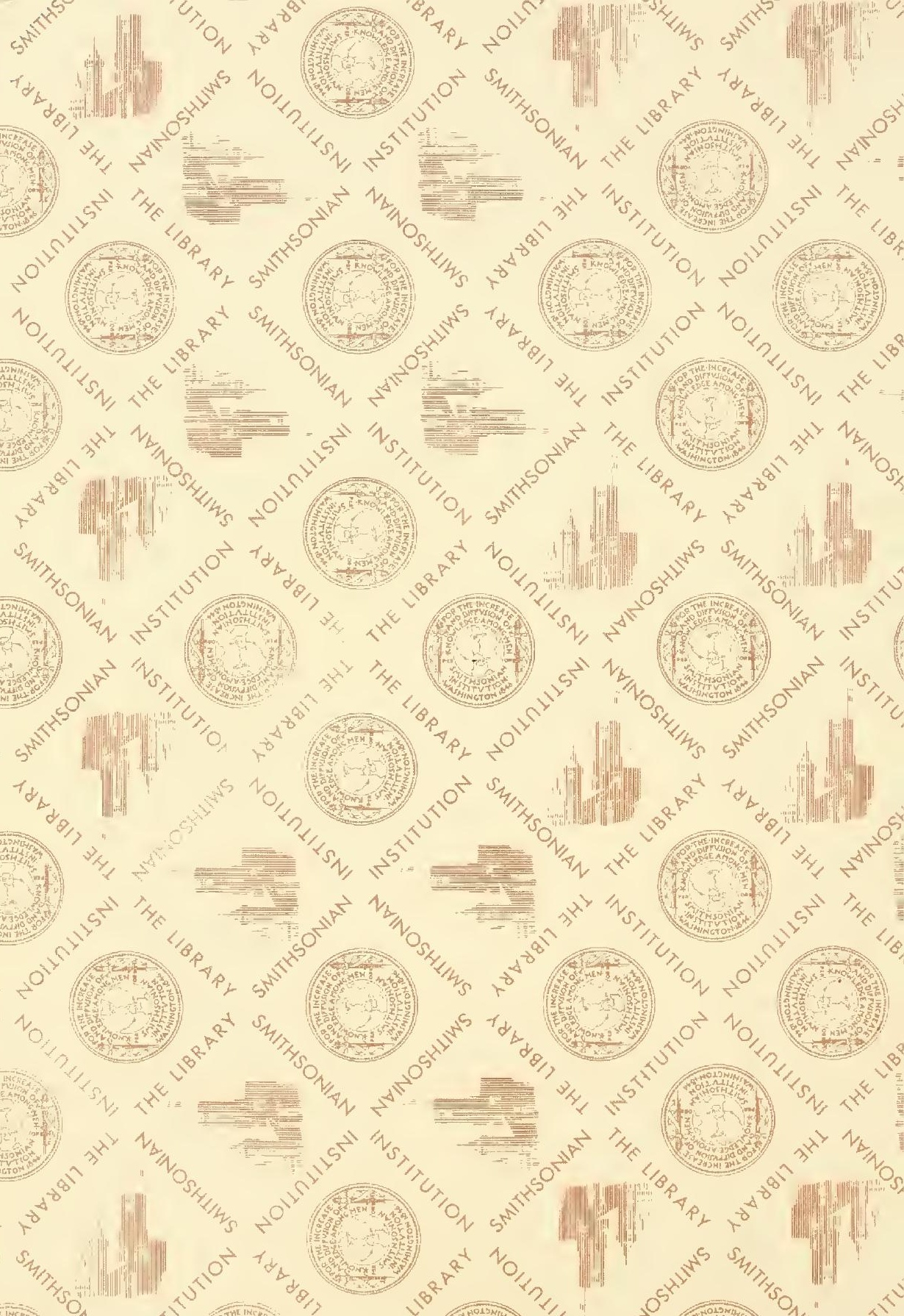




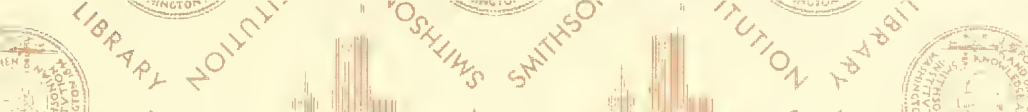

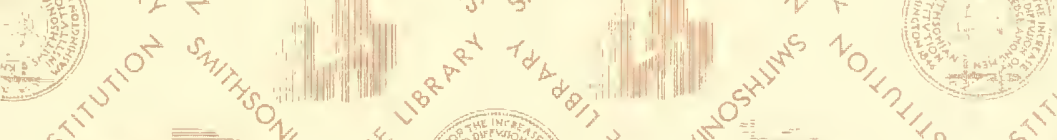

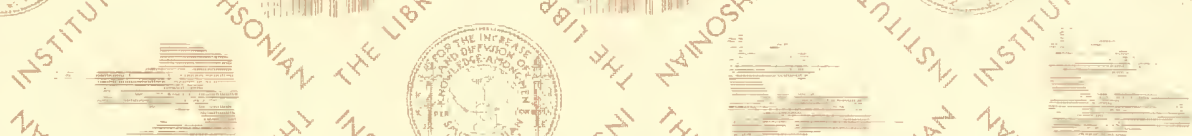

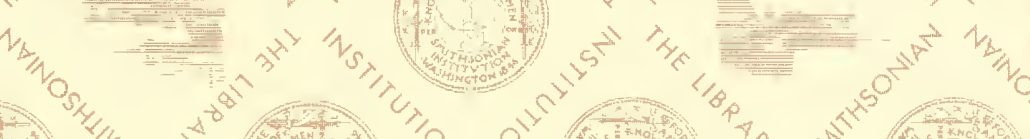

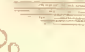

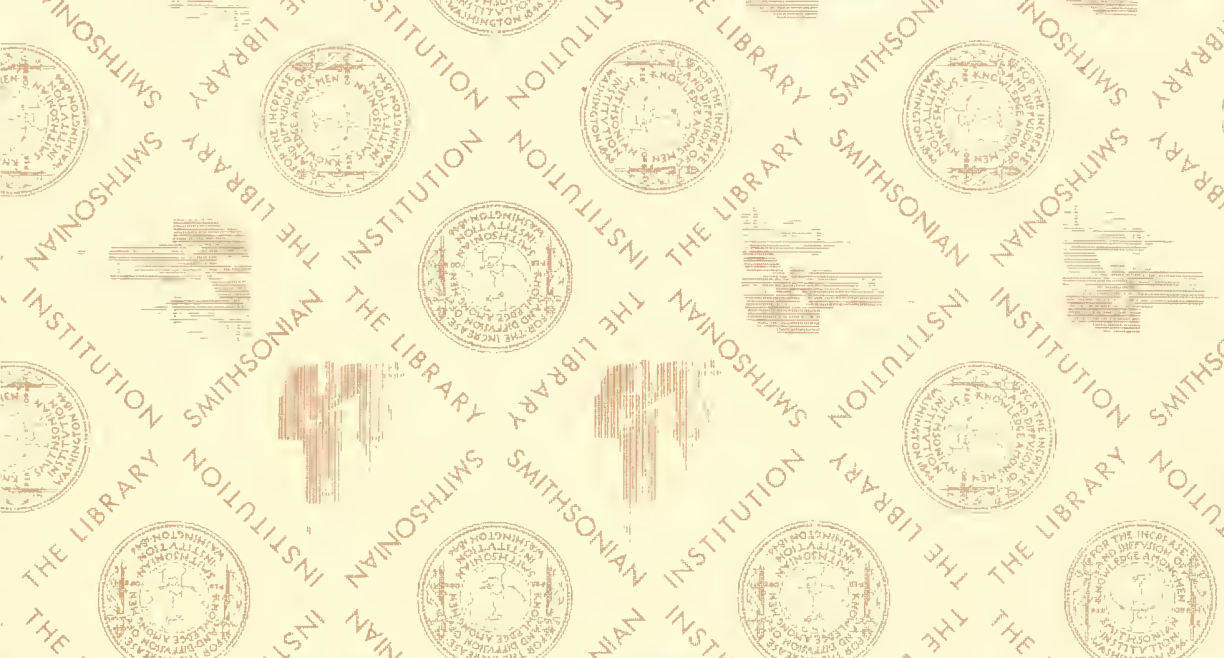

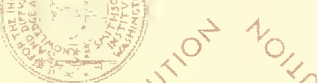<smiles>c1ccc2ccccc2c1</smiles>

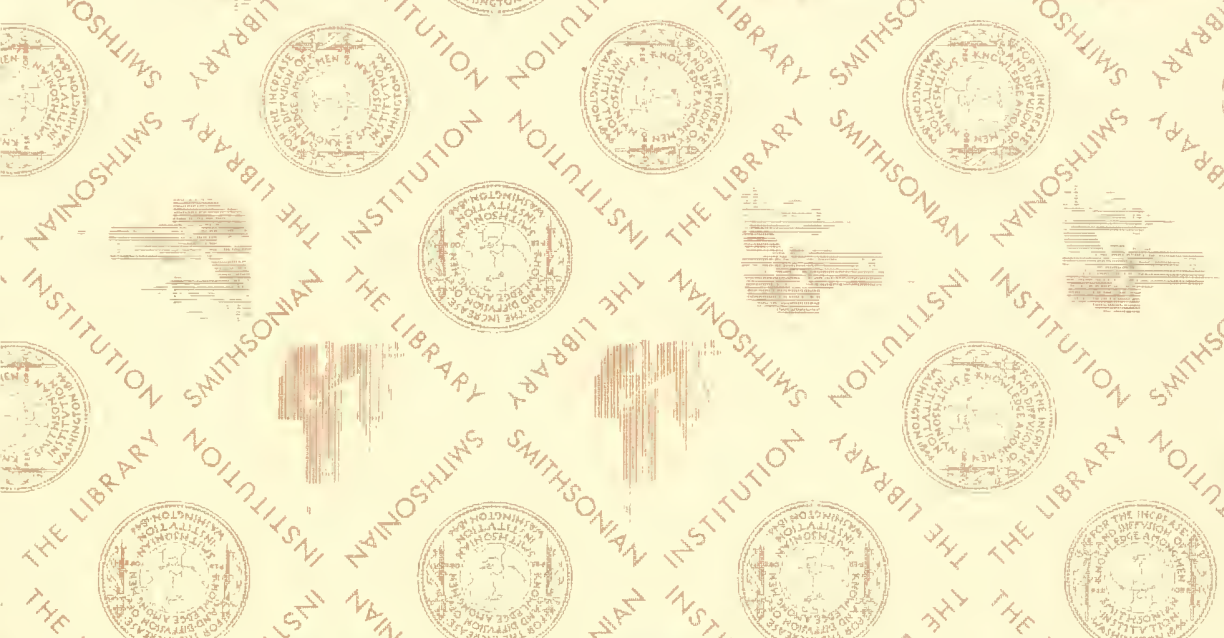

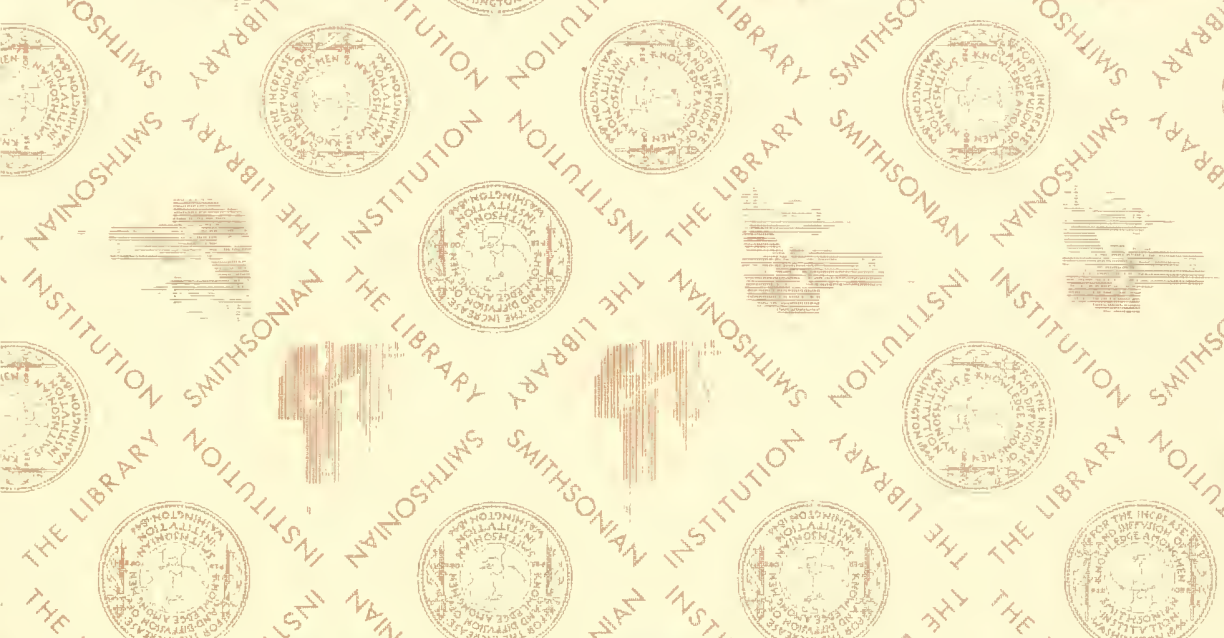

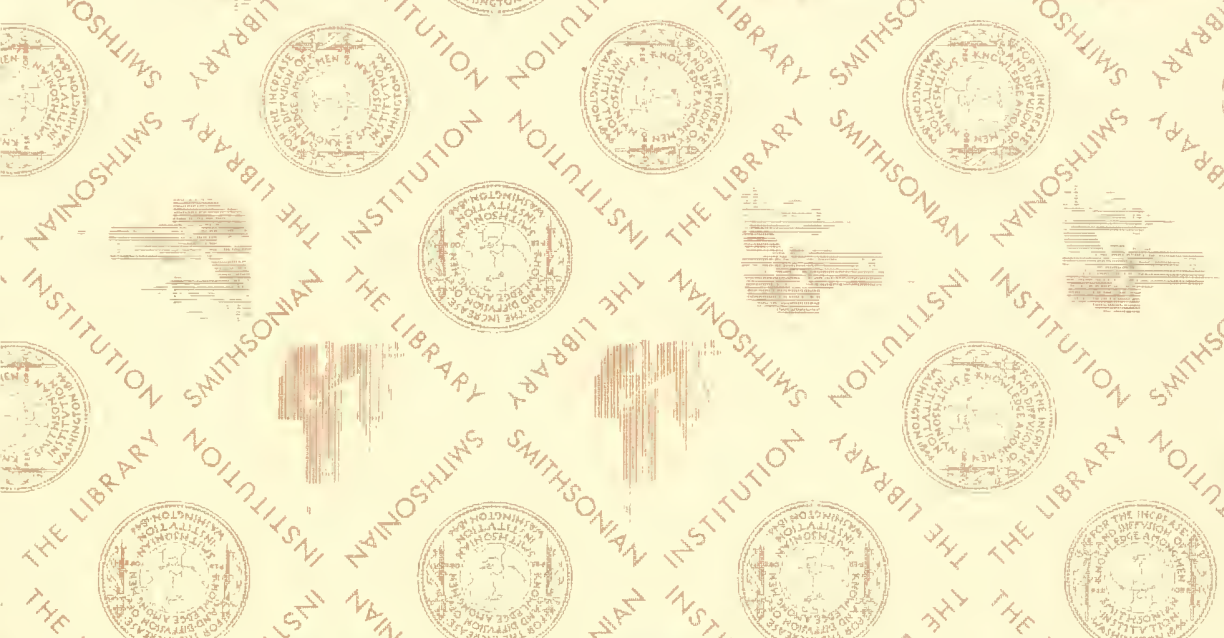

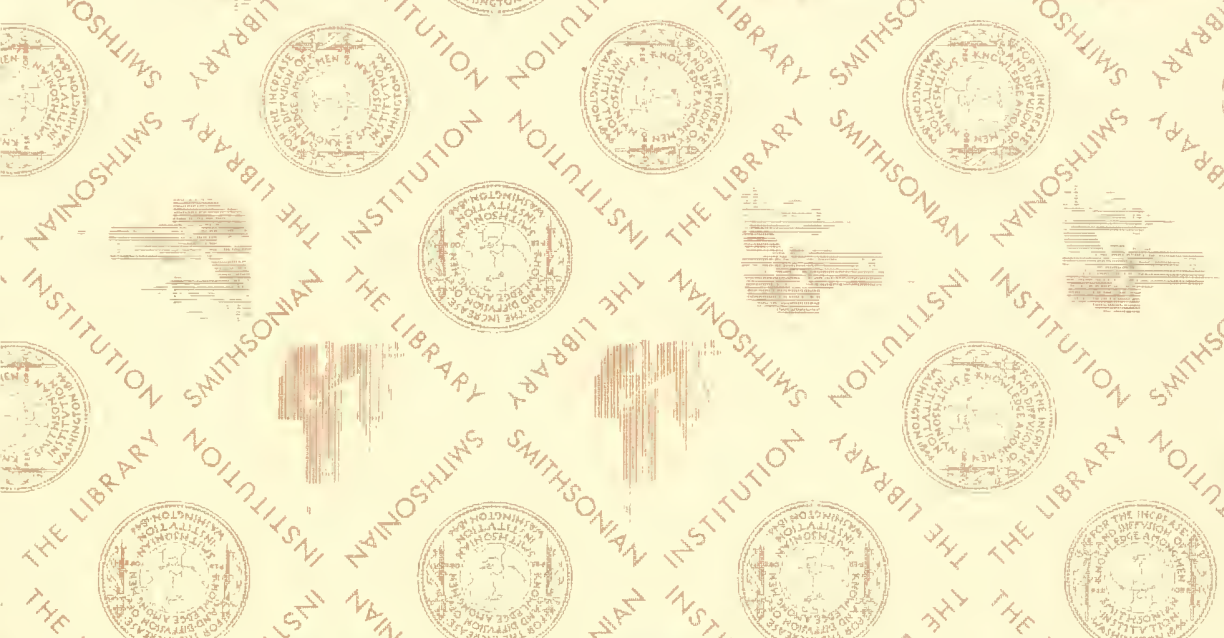

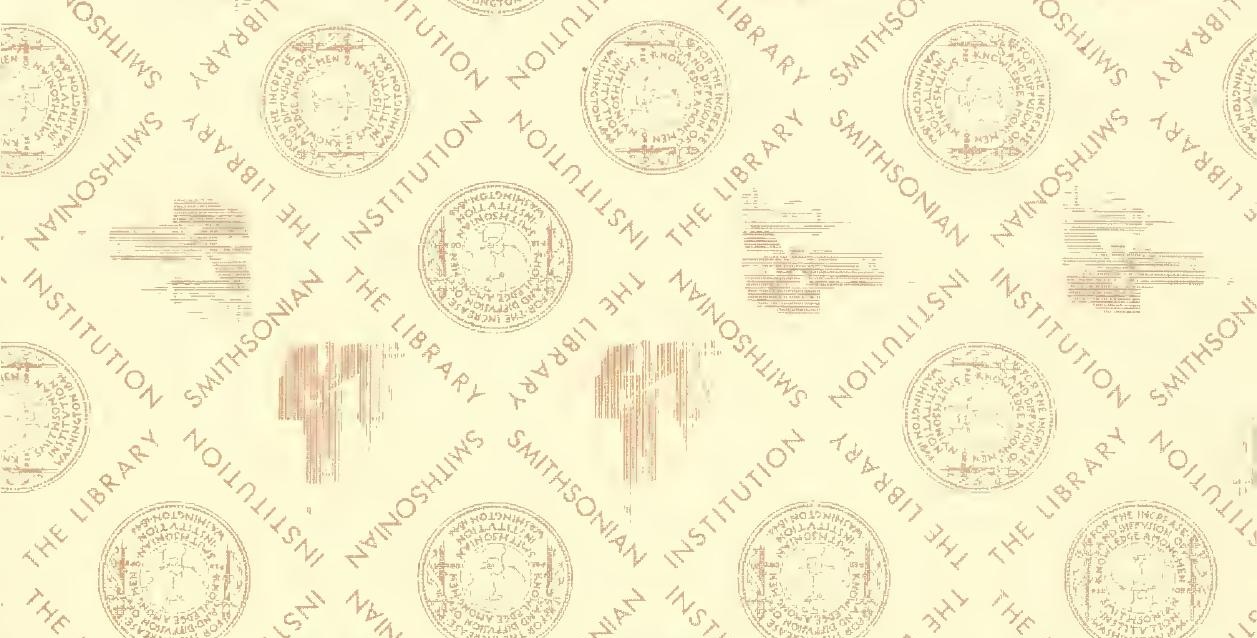

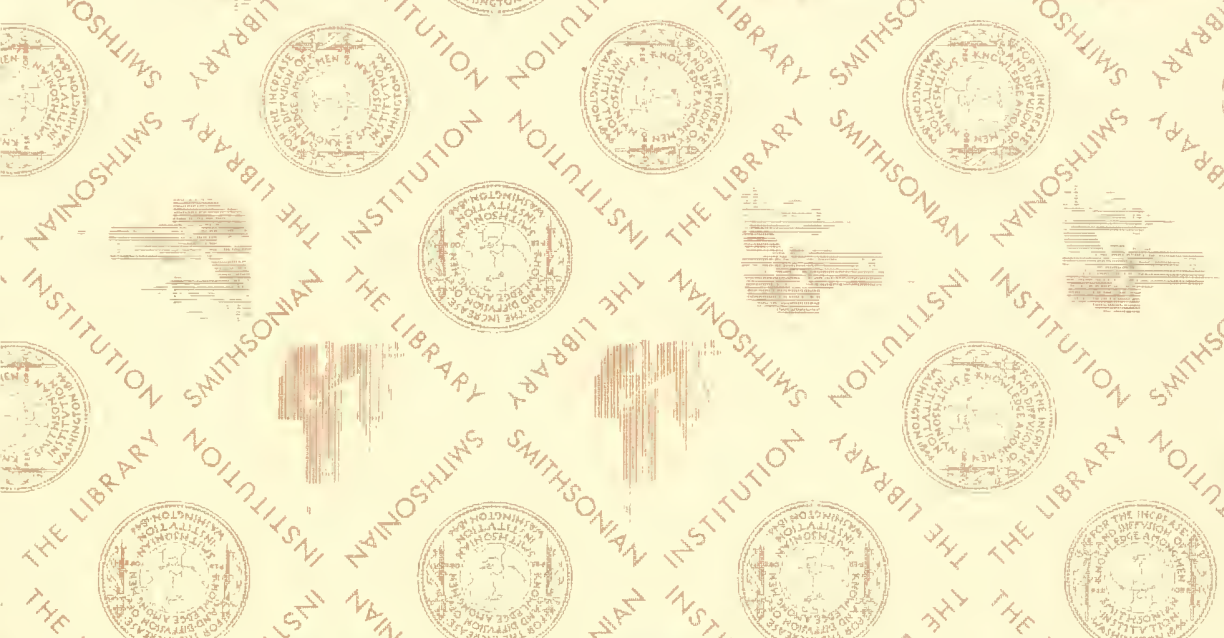

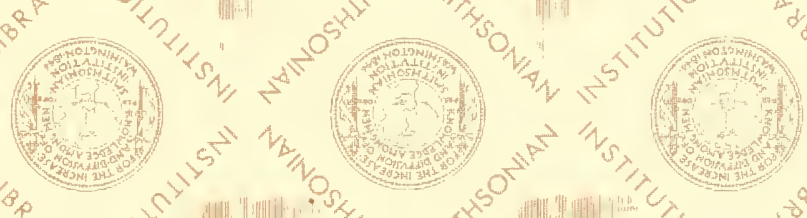

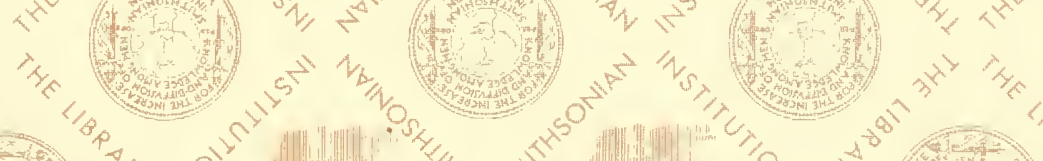

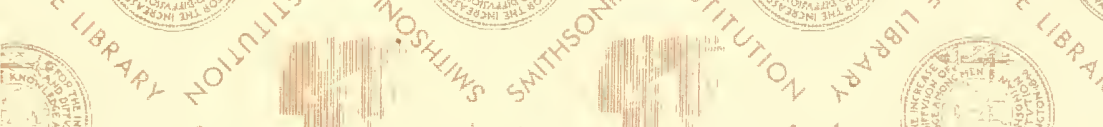

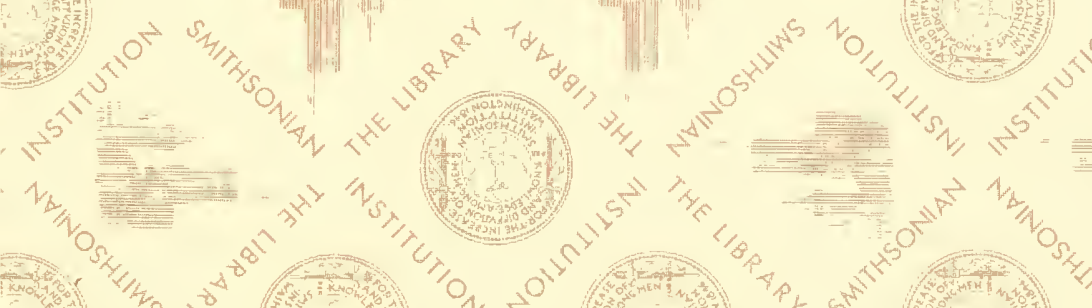

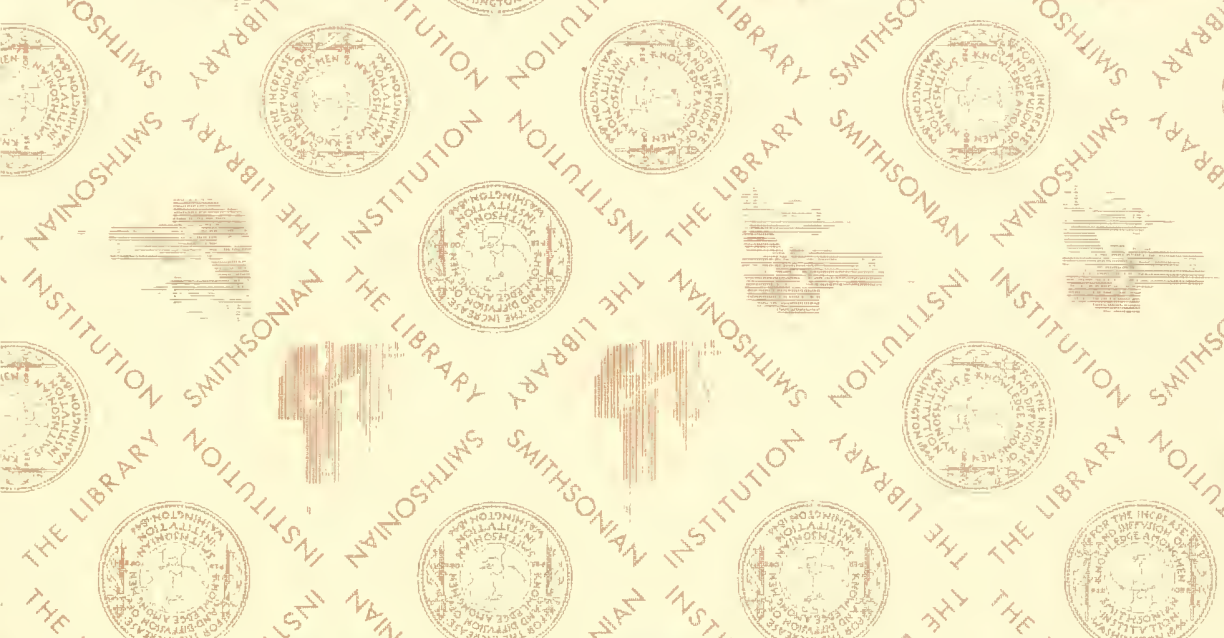
S.

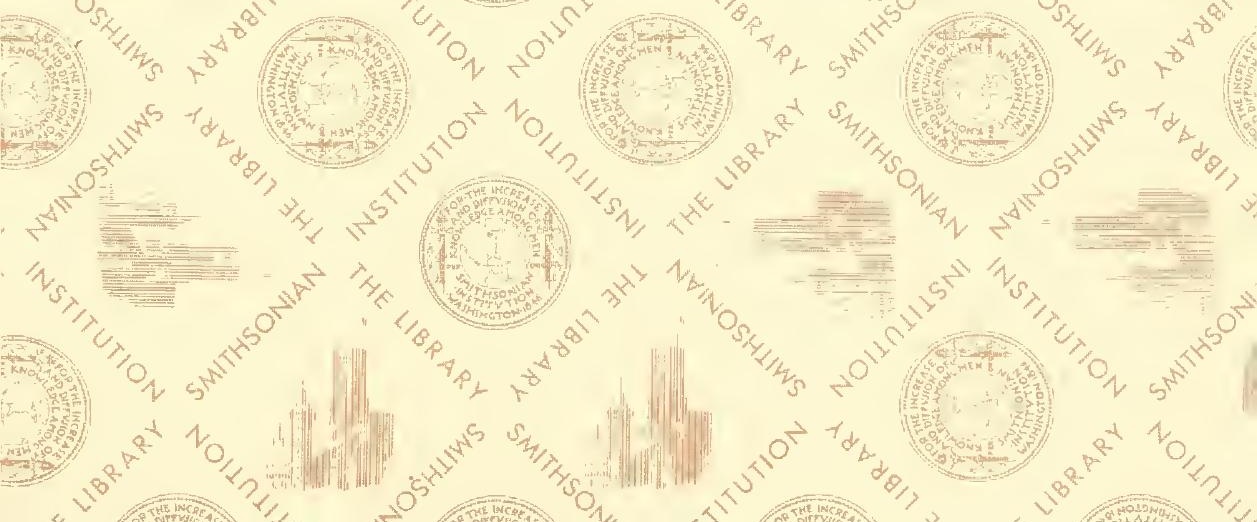


